\title{
Diffractive optics: learning by computer experiments
}

\section{Hartmut Bartelt}

Hartmut Bartelt, "Diffractive optics: learning by computer experiments," Proc. SPIE 3831, Sixth International Conference on Education and Training in Optics and Photonics, (16 June 2000); doi: 10.1117/12.388730

Event: Education and Training in Optics and Photonics (ETOP'99), 1999, Cancun, Mexico 


\title{
Diffractive optics: learning by computer experiments
}

\author{
Hartmut Bartelt*, \\ Institute for Physical High Technology, Jena / Germany
}

\begin{abstract}
Simulation and modeling software is especially useful for visualisation of two-dimensional optical propagation. Based on the software package DigiOpt examples of diffractive optical effects are presented which are difficult to discuss analytically and which have been actual research subjects during the last decades. These examples are intended to demonstrate how modern software technologies can help to gain professional expertise in an efficient way.
\end{abstract}

Keywords: Diffractive optics, computer simulation, education, training

\section{INTRODUCTION}

Modern computer and communication technologies have revolutionized working habits especially in the field of research and development. The use of such technologies for education and training is therefore not only improving the efficiency of learning but also becoming an important part of professional expertise. The use of modern communication technologies allows for example access to:

- on-line publications and journals

- internet as source for modeling software in optics (e.g. http://www.shareware.com)

- electronic data exchange

As in other areas of science modern software allows efficient modeling of complex effects and systems. The availability of high computing power and modestly priced software for educational purposes gives students a good opportunity for interactive learning beyond repetition of preorganized experiments and opens the possibility of small research projects of their own. The field of optics is, due to the two-dimensional effects, a good example how modern computer visualization and presentation methods can useful in education and training. Especially important are:

- visualization of complex optical effects

- simple variations of parameters

- interactive learning

- no limitations due to real optical components

- efficient training and working

As an example of the opportunities given by modern modern software technology we will discuss and present some problems in diffractive optics with the help of a user friendly software DigiOpt developed by Aagedal et al at the University of Karlsruhe / Germany ${ }^{1,2}$. This software was used successfully during the last years in lectures on Fourier optics and holography. The discussed problems were actually research subjects during the last decades and are otherwise difficult to discuss without help of complex experimental work. The examples also show in a good way how the gap between research and education is closing

\section{FEATURES OF DIGIOPT SOFTWARE}

The DigiOpt software was developed at the University of Karlsruhe / Germany. Based on scalar diffraction theory the software models propagation of two-dimensional complex wavefields and includes (fig. 1):

* Correspondence: Email: bartelt@,ipht-jena.de 


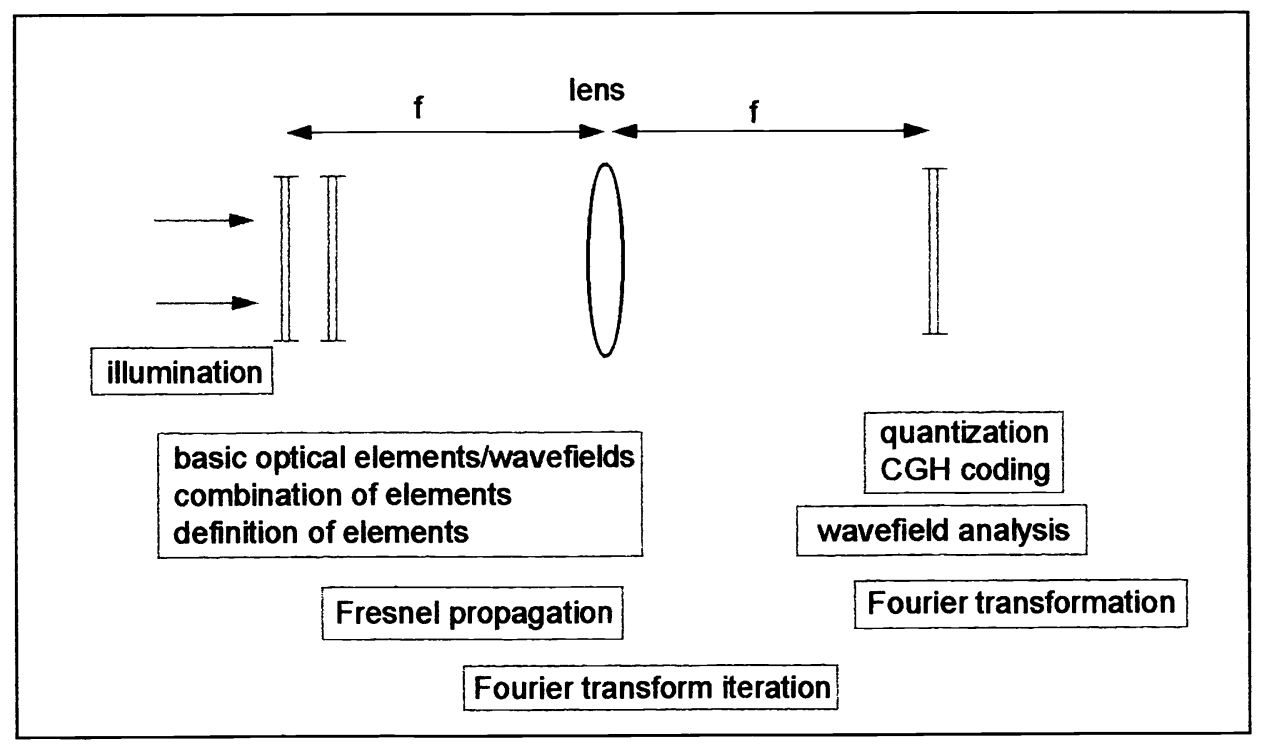

Fig. 1

Features of DigiOpt software

- basic predefined optical elements and user defined optical elements

- Fresnel propagation and Fourier transformation

- combination of wavefronts

- amplitude and phase modifications

- coding of signals including computer generated holograms

A typical example of the program display is shown in fig. 2 for a simple propagation of a wavefield from a mask illuminated with a plane wave.

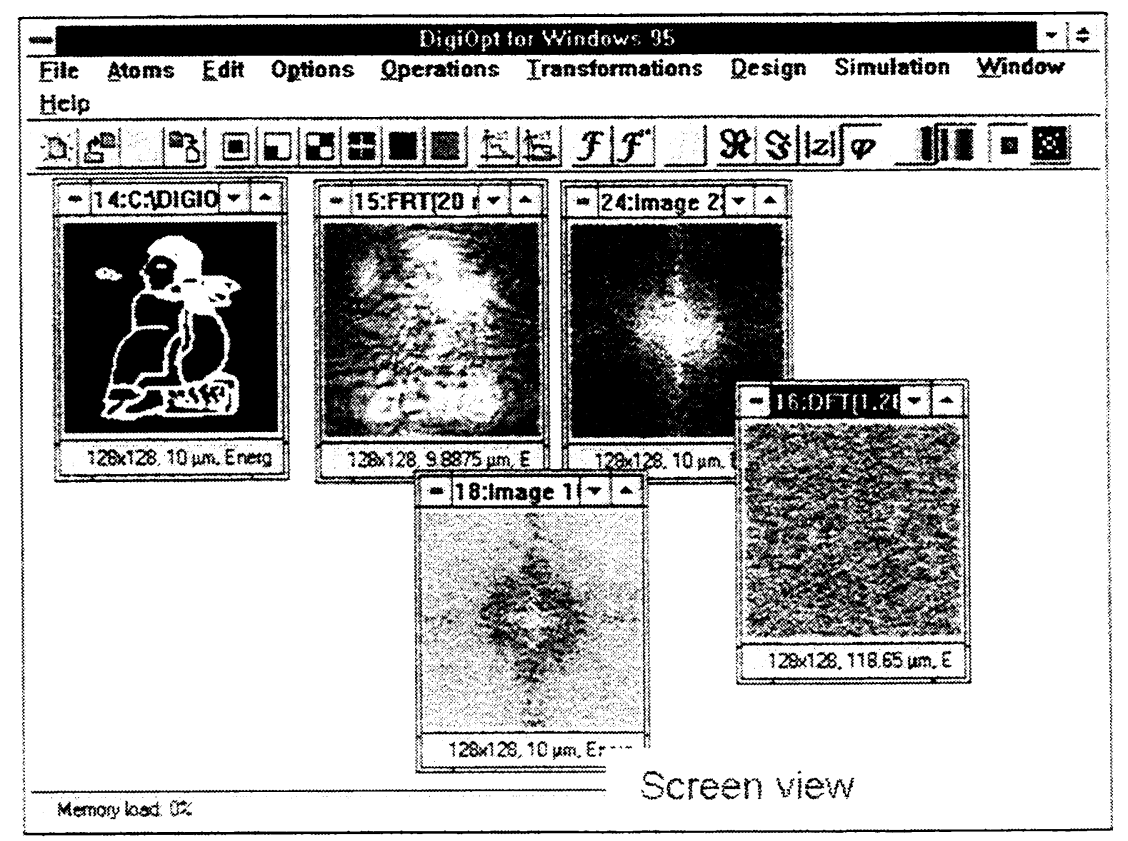

Fig. 2

Typical screen view with computed Fresnel propagation and Fourier transformation 


\section{PROBLEMS IN DIFFRACTIVE OPTICS}

In the following we will discuss and present problems in diffractive optics which are good examples for the use of computer visualization and which are difficult to describe analytically.

The first example refers to the importance of phase and amplitude information in Fourier transforms ${ }^{3}$. In connection with specific computer generated holograms ("Kinoform") the fact of higher importance of phase information has been successfully utilized to generate light efficient phase holograms for display purposes. Fig. 3 presents the visual effects for use of complete complex Fourier plane information, of amplitude only information and of phase only information. It is clearly visible that phase only information is sufficient to recognize the original object (with high pass filtering effect). The amplitude only Fourier space information is not sufficient for a recognizable reconstruction.

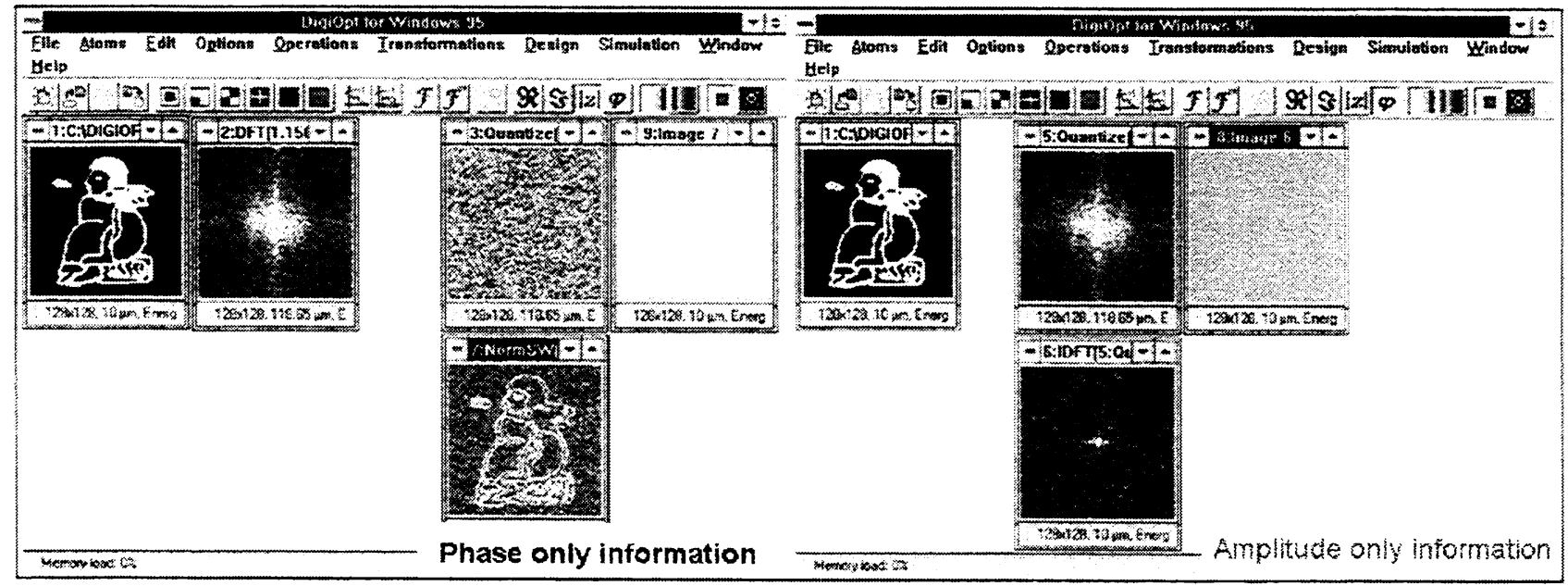

Fig. 3 Importance of Fourier phase and amplitude information, left: object function, Fourier transform, phase of Fourier transform, constant Fourier amplitude, reconstruction from phase only information, right: object function, Fourier transform amplitude, constant Fourier phase, reconstruction from amplitude only information

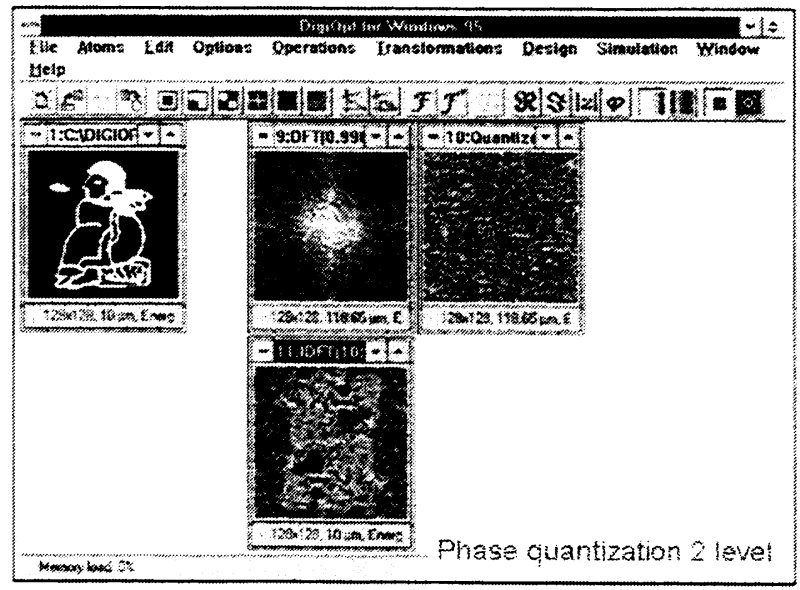

Fig. 4 Effect of binary phase quantisation in Fourier space, top: object, Fourier amplitude, binarized Fourier phase, bottom reconstruction

The next example in fig. 4 visualizes quantization effects in Fourier phase and amplitude ${ }^{4}$. When creating synthetic optical elements it is in most cases unavoidable to introduce quantisation steps in amplitude and/or phase. From grating 
diffraction (corresponding to a Fourier hologram of a single point) it is well known how the number of quantisation steps transforms in a specific theoretical diffraction efficiency. For more complex object functions the effects are difficult to predict analytically. In both figs. 4 and 5 extreme phase quantisation (two levels) in Fourier space has been applied. Strong noise terms and a symmetrical reconstruction are produced. It can be also visualized that a properly shifted object function (fig. 5) avoids overlapping of the noise terms ${ }^{6}$. This result corresponds to the fact that a hermitean object function is equivalent to a real Fourier transform which is therefore not degraded by a two level phase quantisation.

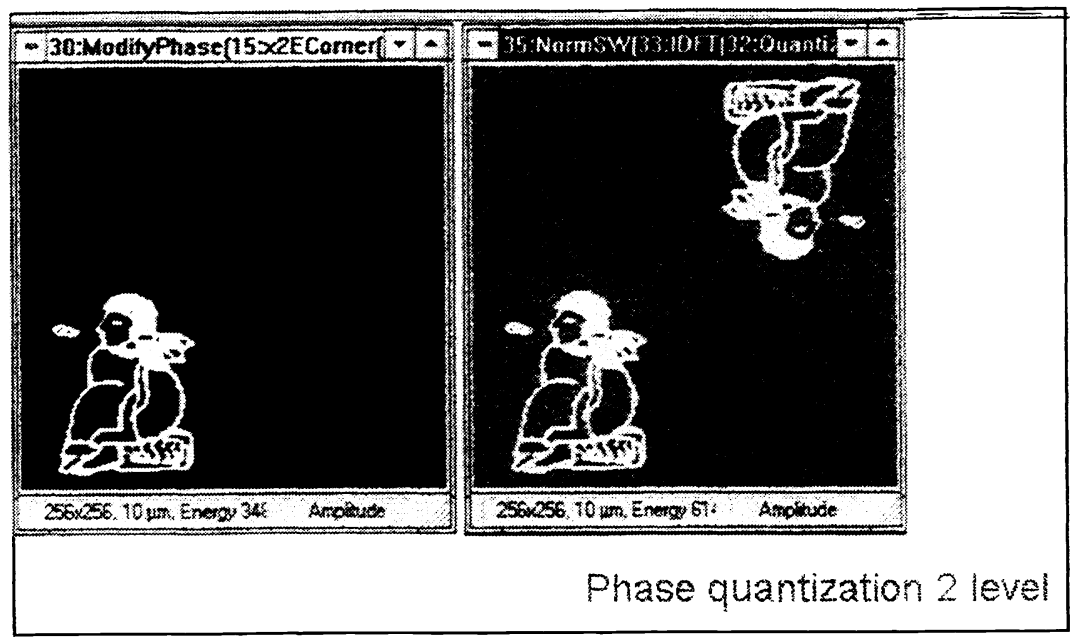

Fig. 5 Shifted object and reconstruction with binarized Fourier phase

In fig. 6 the effect of replications of a pupil mask in the Fourier transform is shown (pupil replication) ${ }^{5}$. Such a replication increases the area of the mask function and can improve energy throughput. Besides concentration of the light at the sampling points the output is equivalent to the object function.

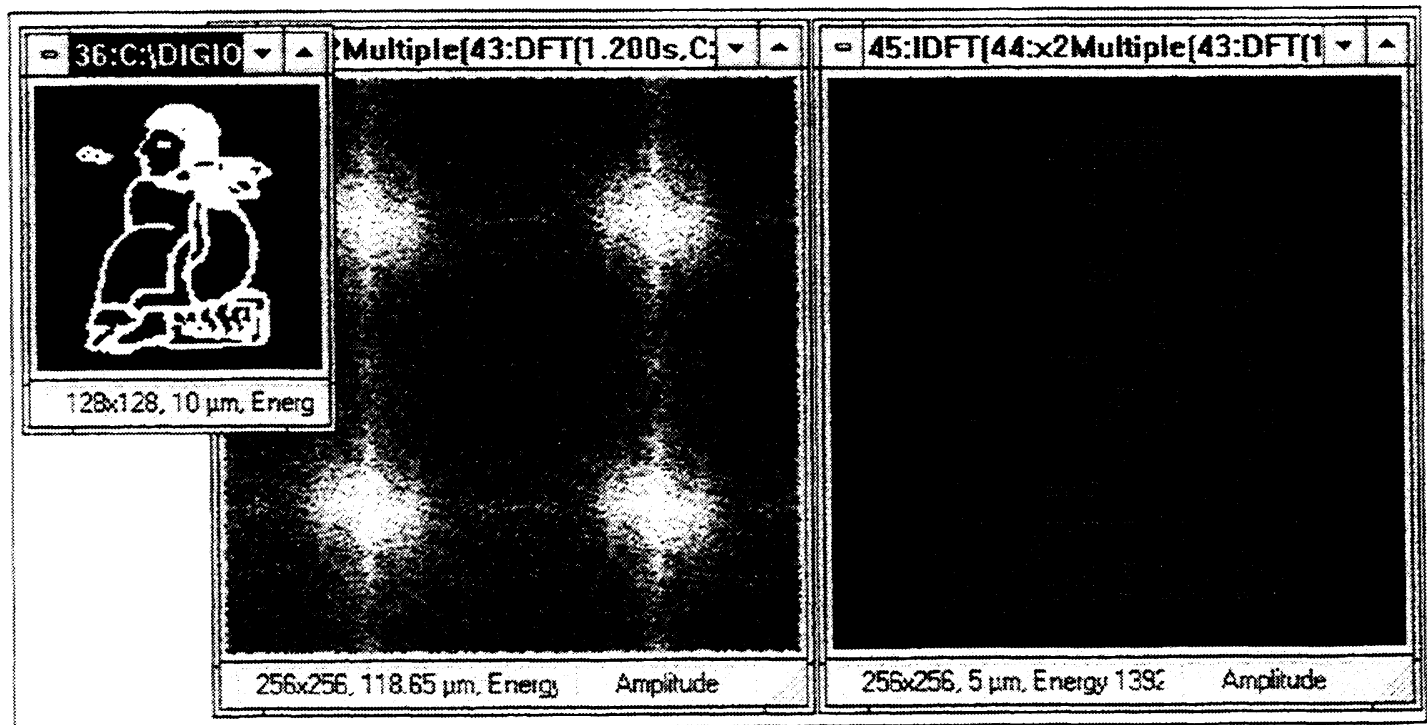

Fig. 6 Effect of 4-fold pupil replication

Complex wavefields are often encoded in the plane of the optical mask as computer generated holograms. Many different coding schemes are possible and it is therefore interesting to investigate the influence of coding schemes on reconstruction quality ${ }^{7}$. In fig. 7 two different types of Lohmann holograms are compared in reconstruction (type I and type II Lohmann hologram). The holograms are of block type and the same number of pixels are used per block. The computed reconstructions show clear differences and visualize the advantages of type III (variable height of openings) over type II (constant height of openings) concerning noise and reconstruction quality. 


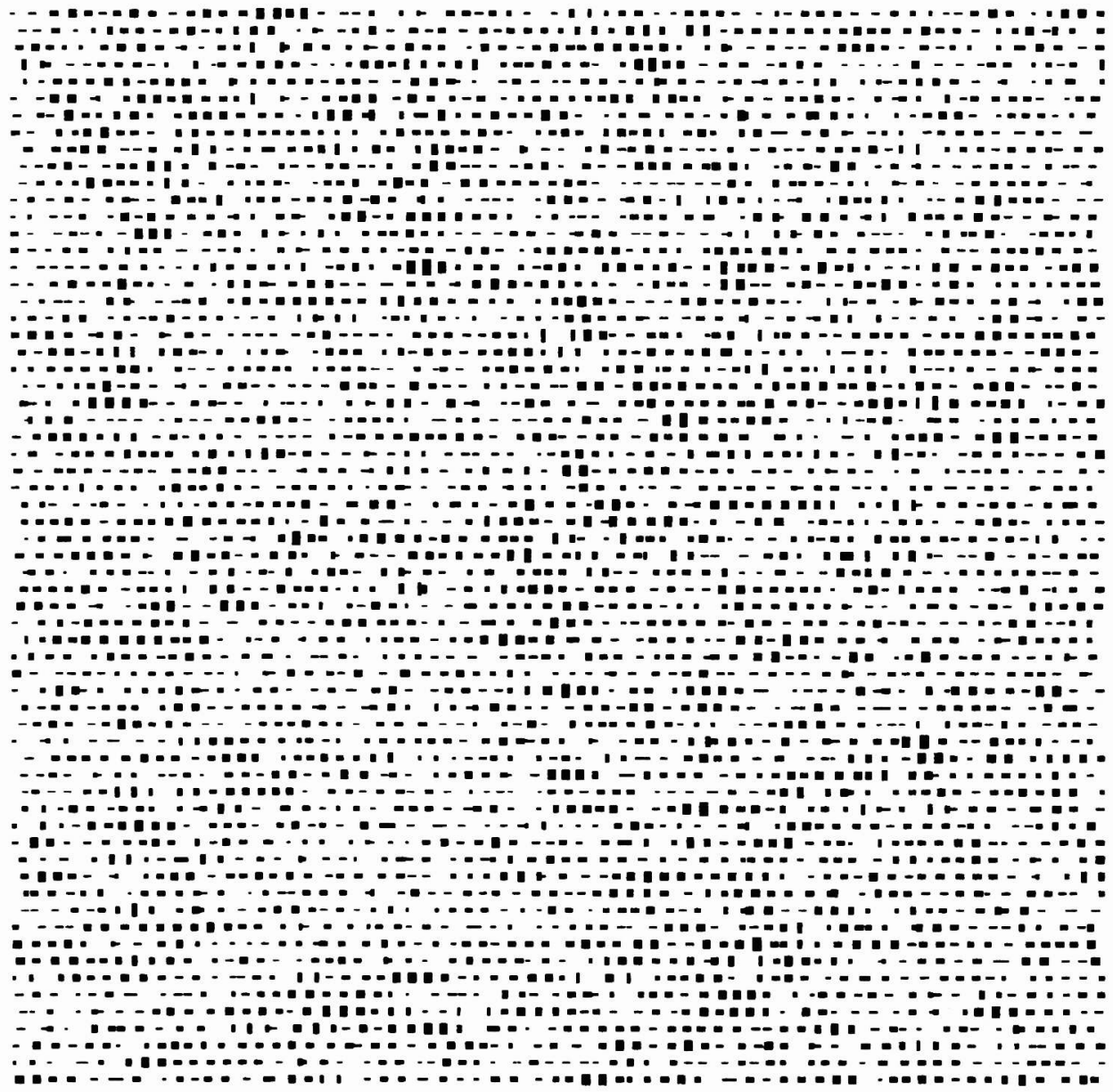

Fig. 7 Computer generated hologram of Lohmann type III, the hologram can be photographed and reconstructed by optical Fourier transformation
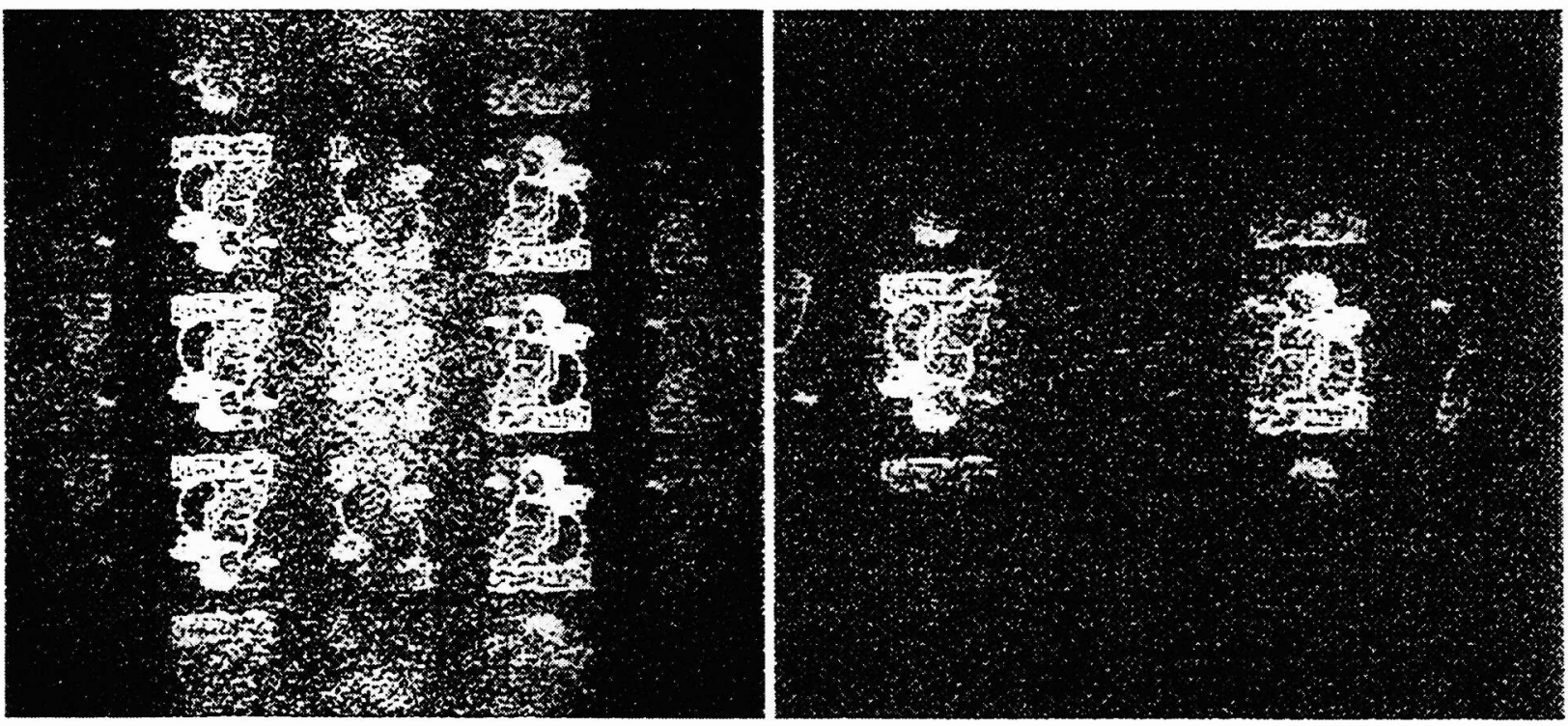

Fig. 8 Simulated reconstruction for the same object function, the same number of block pixels (12x12) but different coding schemes left: Lohmann type III with vanable height of openings (from hologram in fig. 7), right: Lohmann type II with constant height of openings, the better reconstruction quality of type III is due to better use of the quantisation degrees of freedom in the hologram 
The iterative Fourier algorithm is a method often used to optimize reconstruction quality from phase only Fourier holograms ${ }^{8}$. The improvement of reconstruction quality versus number of iterations is effectively visualized in fig. 9.

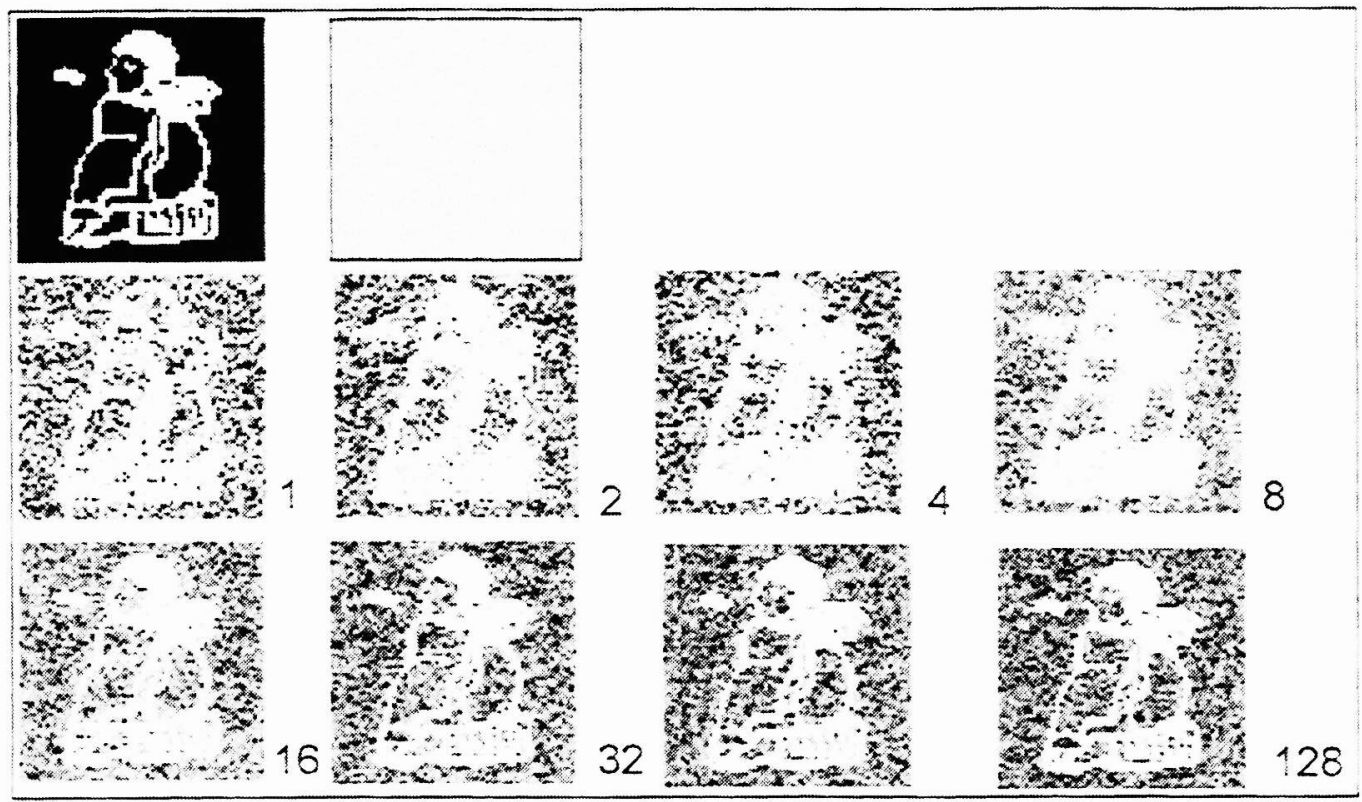

Fig. 9 Visualisation of iterative Fourier algorithm iteration, top: object function, phase only mask, bottom: phase only mask / constant amplitude reconstruction for $1,2,4,8,16,32,64$, and 128 iterations

The final example in figs. 10 and 11 refers to Fresnel propagation effects. Usually, propagation effects due to diffraction result in a considerable change of the wavefield. This can be interpreted as a limited depth of focus. For many practical applications it would be desirable to increase this depth of focus. There are specific wavefields known which correspond to so-called undiffracted beams and which do not change by propagation. Using the optical modeling software we can approximate a "slowly diffracting beam" for a general object. For this purpose several optical mask functions are computed which would reconstruct the object function in different depth planes. The data of these functions are summarized or averaged to give the final optical mask. The reconstruction result shown in figs. 10 and 11 presents the effect of conventional propagation and for propagation from the synthesized mask. The result shows how a wavefield with slow depth variation can be achieved under constraints of wave propagation.

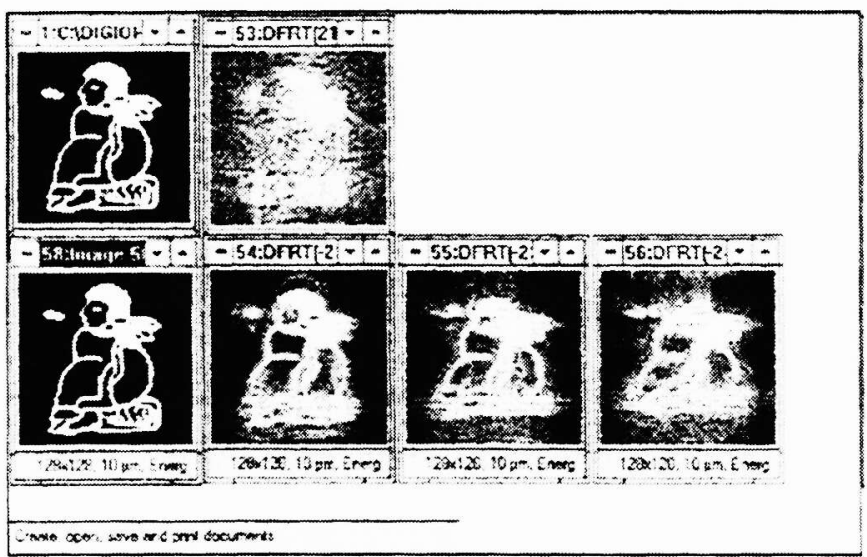

Fig. 10 Wavefield propagation in space, top: object and mask function, bottom: wavefield $21 \mathrm{~mm}, 22 \mathrm{~mm}, 23 \mathrm{~mm}$ and $24 \mathrm{~mm}$ behind mask function

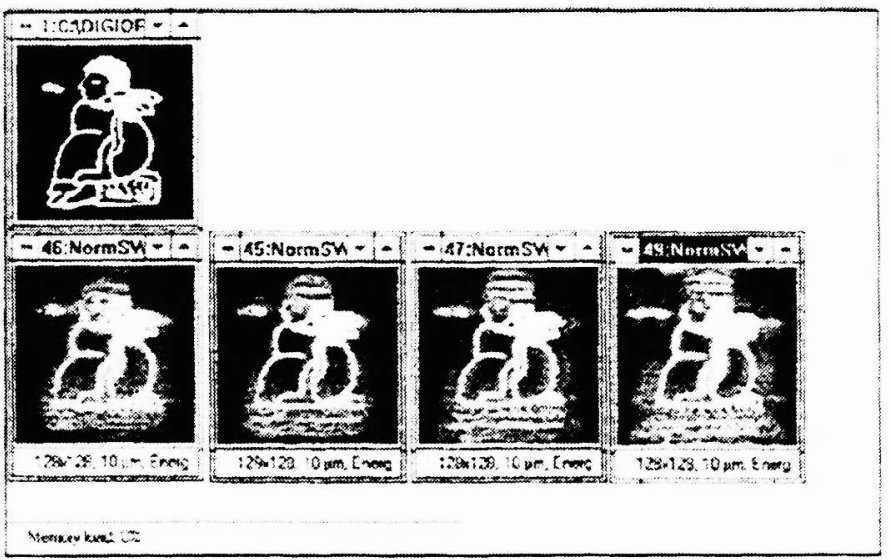

Fig. 11 Wavefield propagation for slowly diffracting wave, top: object function, bottom: wavefield $21 \mathrm{~mm}, 22 \mathrm{~mm}$, $23 \mathrm{~mm}$ and $24 \mathrm{~mm}$ behind averaged mask function 


\section{CONCLUSIONS}

Computer physics is an emerging area of interest which is useful for training and education especially in optics. Using appropriate modeling software not only motivates by achieving direct results but also allows flexible solutions to different problems. Developing and improving such software using new computing technology will be therefore an attractive task for the future.

\section{ACKNOWLEDGEMENT}

The author would like to thank very much A. Aagedal and T. Schmid for their cooperation and the possibility of using the DigiOpt software.

\section{REFERENCES}

1. H. Agedal, T. Beth, H. Schwarzer, S. Teiwes, "Design of paraxial diffractive elements with the computer-aided design CAD system DigiOpt", SPIE Vol. 2404, pp. 50-58, 1995

2. H. Aagedal, S. Egner, M. Schmid, T. Beth, "Important aspects of numerical implementation of wave propagation between parallel planes demonstrated with the CAD system DigiOpt", EOS Topical Meeting Digest Series Vol. 12, p. 162,1997

3. L.B. Lesem, P.M. Hirsch, J.A. Jordan, IBM J. Res. Develop.13,pp. 150, 1969

4. W.J. Dallas, Computer generated holograms, in : The computer in optical research / Ed.: B.R. Frieden, Springer, New York, 1980

5. B. Braunecker, R. Hauck, A.W. Lohmann, Appl. Opt. 18, p. 2786, 1979

6. W.J. Dallas, Appl. Opt. 10, pp. 673-679, 1970

7. P. Hariharan, Optical Holography, Cambridge University Press, p. 163, 1996

8. J.R. Fienup, SPIE Vol. 373, p. 147, 1981 